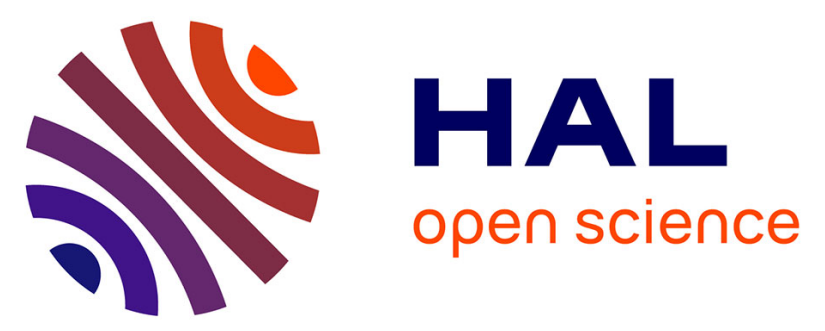

\title{
New planar nano-gauge detection microphone: Analytical and numerical acoustic modeling
}

Cécile Guianvarc'H, Thierry Verdot, Jaroslaw Czarny, Emmanuel Redon, Kerem Ege, Jean-Louis Guyader, Arnaud Walther, Philippe Robert

\section{To cite this version:}

Cécile Guianvarc'H, Thierry Verdot, Jaroslaw Czarny, Emmanuel Redon, Kerem Ege, et al.. New planar nano-gauge detection microphone: Analytical and numerical acoustic modeling. 21st International Congress on Acoustics (ICA 2013), Jun 2013, Montréal, Canada. 2pEAa2., 10.1121/1.4800608 . hal-00823979

\section{HAL Id: hal-00823979 \\ https://hal.science/hal-00823979}

Submitted on 20 May 2016

HAL is a multi-disciplinary open access archive for the deposit and dissemination of scientific research documents, whether they are published or not. The documents may come from teaching and research institutions in France or abroad, or from public or private research centers.
L'archive ouverte pluridisciplinaire HAL, est destinée au dépôt et à la diffusion de documents scientifiques de niveau recherche, publiés ou non, émanant des établissements d'enseignement et de recherche français ou étrangers, des laboratoires publics ou privés. 
New planar nano-gauge detection microphone: analytical and numerical acoustic modeling

Cécile Guianvarc' $h{ }^{1}$, Thierry Verdot ${ }^{1}$, Jaroslaw Czarny ${ }^{1,2}$, Emmanuel Redon ${ }^{1}$, Kerem Ege ${ }^{1}$, Jean-Louis Guyader ${ }^{1}$, Arnaud Walther ${ }^{2}$ and Philippe Robert ${ }^{2}$

1) Laboratoire Vibrations Acoustique, INSA-Lyon, 25 bis Avenue Jean Capelle, F-69621 Villeurbanne Cedex, France

2) Univ. Grenoble Alpes, F-38000 Grenoble. CEA, LETI, MINATEC Campus, F-38054 Grenoble, France 


\section{INTRODUCTION}

Microphones miniaturization is of great interest for several fields, such as medical applications (audio implants), or consumer electronics (cell phones). One of the most important limitation is the decrease of the microphones performances (sensitivity, resolution) with severe miniaturization. In this field, a new concept of microphones developed by CEA-LETI, which uses membranes moving in the plane of the substrate and inducing strain on piezoresistive $\mathrm{Si}$ nano-gauges (M\& NEMS technology), seems promising for its miniaturization potential without significant decrease of sensitivity. The M\& NEMS concept of piezo-resistive detection with $\mathrm{Si}$ nano-gauges has already been demonstrated on inertial sensors such as gyroscopes [1], and results obtained on these sensors confirm the miniaturization potential of this new principle.

The optimization of such planar piezo-resistive microphone requires a deep understanding of its acoustic and vibroacoustic behavior. Regarding the small dimensions of the slits $(1-100 \mu \mathrm{m})$ and the sharp discontinuities in the microphones structure, viscous and thermal effects in the boundary layers, their coupling to the membranes vibroacoustic behavior, and even to turbulent perturbations, should be taken into account with high accuracy in device modeling.

Analytical solutions to the Kirchhoff equations in viscothermal fluids [2] presenting a good agreement with experimental results have already been suggested. However, the solutions were found for acoustic devices with simpler shapes [3] or higher dimensions [4]. Regarding the complex shape, small dimensions and strong fluid/structure couplings in this microphone, numerical modeling procedure has to precisely define all the phenomena in the microphone.

During the last few years, several numerical modelings that use boundary element methods and finite element methods (BEM/FEM) have been carried out $[5,6,7,8]$. Those models describe acoustic fields and/or vibroacoustic membranes and plates behaviors that are coupled to viscous and thermal diffusive fields in the boundary layers. All the studies rely on the fundamental equations of acoustics in dissipative fluids (Navier-Stokes, conservation of mass, and Fourier equations), but because of the choice of the variables for the acoustic problem, and on some simplifying hypothesis, they lead to different formulations.

The aim of the present work is to provide accurate and adapted analytical and numerical (FEM) models able to gather all these effects in a consistent manner.

\section{Planar Piezo-resistive microphone, PRESSURE SENSitivity}

\subsection{Basic principle and fabrication}

A schematic view of the planar microphone with four membranes is shown on Figure 1. Each membrane is made of a rigid beam able to move within the plane of the substrate ; it is anchored at one end with a hinge and free at the other end. A Si gauge with nanometer size section (down to $250 \times 250 \mathrm{~nm}^{2}$ ) is suspended between the moving beam and an anchor. The microphone's input can be either the set of two top slits, or the set of three bottom slits (Fig. 1a).

The membranes are coupled to the input acoustic field by "large" top or bottom input slits (width $100 \mu \mathrm{m}$, length $300 \mu \mathrm{m}$ ), also connected to "large" output slits by the four membranes and eight "thin" and "short" slits above and under the membranes (width 1-2 $\mu \mathrm{m}$, length $2 \mu \mathrm{m}$ ).

As shown on Figure 2, for practical use, the microphone and its electronics (designed by the Institut Matériaux Microélectronique Nanosciences de Provence, IM2NP Marseille) are enclosed together in a package preventing them from external pollution. Depending on the position of the input hole in the package for the acoustic wave and on the side on which we bond the connections on the MEMS, the microphone can be in top-port configuration (Fig. 2a) or bottom-port configuration (Fig. 2b). 


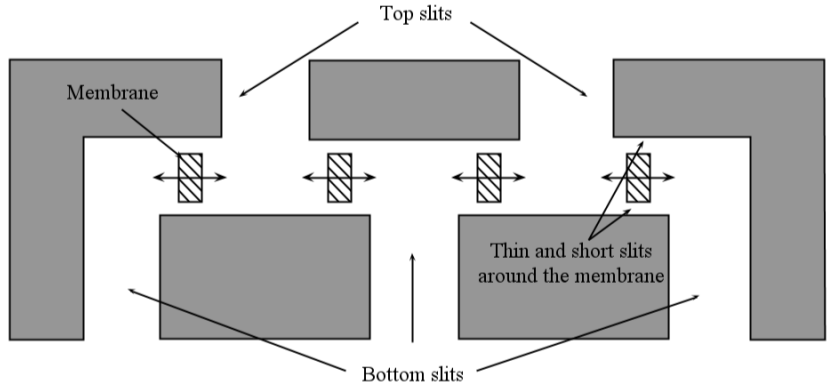

(a)

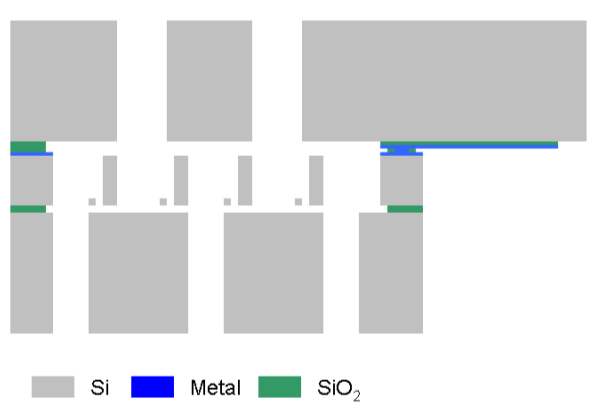

(b)

FigURE 1: Planar piezo-resistive microphone: (a) schematic view, (b) side view of the fabrications process.

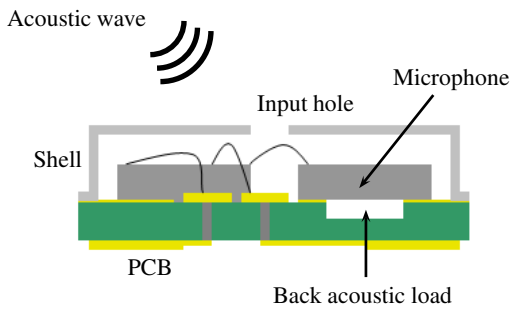

(a) Top-port mounting

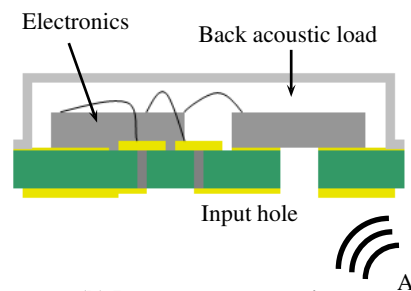

(b) Bottom-port mounting

FIGURE 2: Suggested package configuration for a planar piezo-resistive microphone.

In the top-port configuration, incident waves arrive to the microphone's input through the hole in the shell of the package, and the cavity formed by the package (Helmholtz resonator), the bottom slits being loaded acoustically by a small cavity in the printed circuit board (PCB). On the other hand, in the bottom-port configuration, the incident waves arrive to the microphone's input through a simple hole in the PCB, the top slits being loaded by the volume of the package.

The technological fabrication is carried out in clean room with process from micro-electronics and starts with SOI substrate with $1 \mu \mathrm{m}$ buried oxide and $0.25 \mu \mathrm{m}$ Si top layer. Nano-gauges are defined by DUV lithography and etched by RIE. Then a $1.5 \mu \mathrm{m}$ thick oxide is deposited in order to protect the gauges. Lithography and RIE etching of this oxide only maintain this layer on top of nano-gauges. $10 \mu \mathrm{m}$ thick mono-crystalline silicon is then grown by epitaxy and MEMS structure is defined by DRIE. A cap with contact redistribution layer is bonded on top of the first wafer with eutectic bonding. Handle and cap wafers are then thinned and etched with DRIE in order to open top and bottom slits. The mechanical structure is eventually released with vapor HF etching. A side view of the stack is shown on Figure 1b.

\subsection{Piezo-resistive transduction equations}

The piezo-resistive transduction in the microphone is realized by a nano-gauge with cross section $S_{g}$. Moving beam of length $\ell$ generates longitudinal strain in a gauge. The beam is attached to a micro-hinge at one side while the opposite side is free. The acoustic pressure $p_{m}(t)$ acting on the surface of a beam $S_{m}$ produces a force $f(t)=p_{m}(t) S_{m}$. The mechanical system here benefits of the lever effect, thus one can calculate the force $f_{g}(t)$ generated in a nano-gauge placed at a distance $d_{g}$ from the rotation axis in the following manner:

$$
\frac{f_{g}(t)}{S_{g}}=\frac{f(t) \ell}{S_{g} 2 d_{g}}=\frac{p_{m}(t) S_{m} \ell}{S_{g} 2 d_{g}} .
$$




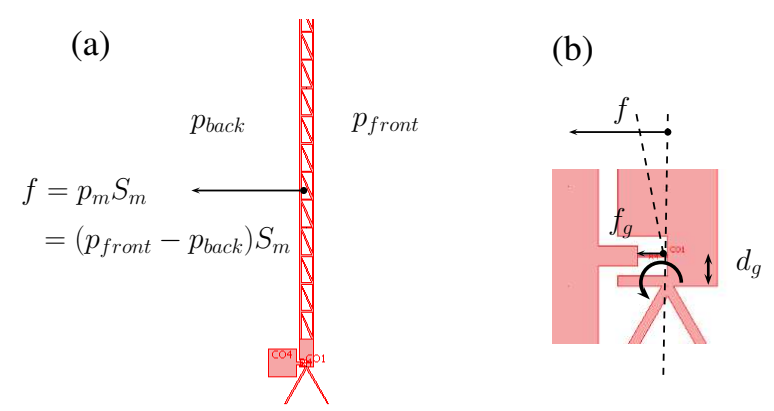

FIGURE 3: (a) Forces applied to a membrane coupled to a nano-gauge, (b) Resulting force applied to the nano-gauge.

Consequently, the stress variation $f_{g}(t) / S_{g}$ loading the piezo-resistive nano-gauge in compression and tension generates a variation in the total electric resistance $R(t)=R_{0}+r(t)$ measured between the ends of the gauge, and related to strain through piezo-resistive coefficient $\Pi_{P Z R}$. The electric tension $U(t)$ between the ends of the gauge, when imposing a constant current $I_{0}$ through it, is then given by

$$
U(t)=R(t) I_{0}=\left(1+\frac{r(t)}{R_{0}}\right) R_{0} I_{0}=\left(1+\Pi_{P Z R} \frac{p_{m}(t) S_{m} \ell}{S_{g} 2 d_{g}}\right) R_{0} I_{0} .
$$

We thus benefit from 2 amplification mechanisms: a lever effect ( $\ell / 2 d_{g}$ factor) and the strain concentration in the nanogauge due to its nanometric section $S_{g}$.

\subsection{Pressure sensitivity of a planar gauge-detection microphone}

The microphone profits of four strain nano-gauges arranged into a Wheatstone bridge that is supplied by a constant voltage $U_{\text {ext }}$ (Fig. 4), in order to measure the resistance variations.
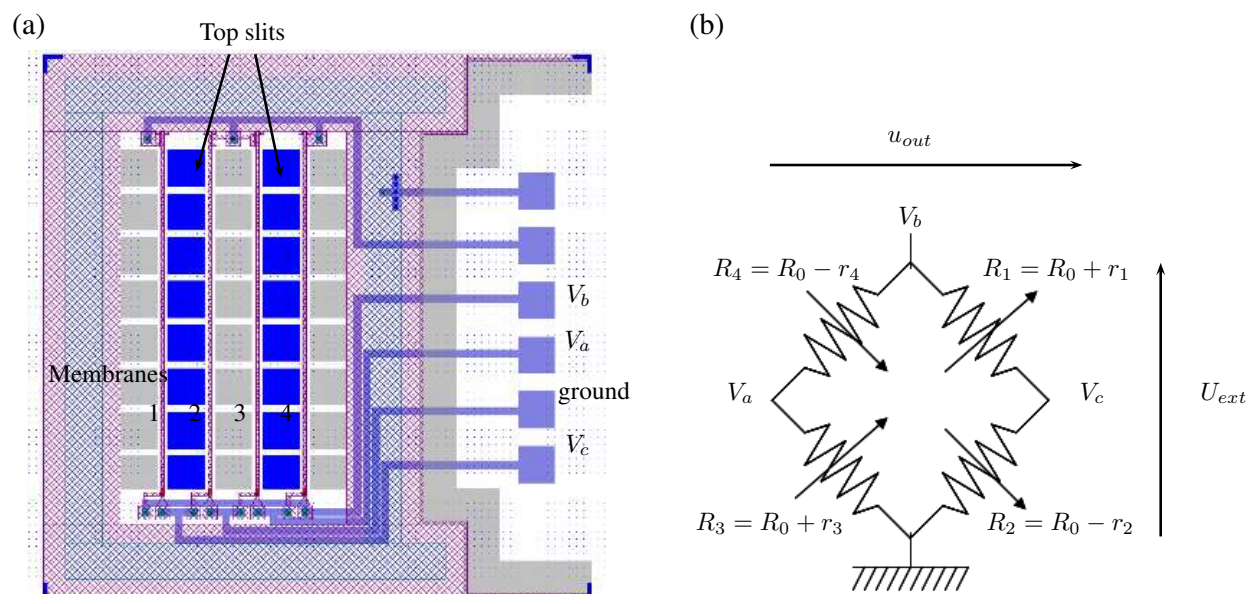

FigURE 4: (a) Top view of a planar gauge-detection microphone, (b) Wheatstone bridge related to the microphone.

Finally, the pressure sensitivity $M_{p}$ of the microphone, defined as the ratio of the open-circuit voltage $u_{\text {out }}$ measured at the output of the Wheatstone bridge, to the acoustic pressure $p_{i n}$ at the input of the microphone, is written as

$$
M_{p}=\frac{u_{\text {out }}}{p_{\text {in }}}=\frac{U_{\text {ext }}}{4 R_{0}} \frac{\Pi_{P Z R} S_{m} \ell}{S_{g} 2 d_{g}} \sum_{i=1}^{4} \frac{p_{m i}}{p_{\text {in }}},
$$

where the pressures applied to each membrane are denoted $p_{m i}$. 


\section{Modeling of The ACOUStical Behavior OF THE MiCROPHONE}

\subsection{Linearized fundamental equations of acoustics}

The microphone (Fig. 1) is used in atmospheric conditions (air at rest). The parameters specifying the gas properties are the static pressure $P_{0}$, and temperature $T_{0}$, the density $\rho_{0}$, the shear viscosity coefficient $\mu$, the bulk viscosity coefficient $\eta$, the coefficient of thermal conductivity $\lambda$, the specific heat coefficient at constant pressure and constant volume per unit of mass $C_{P}$ and $C_{V}$ respectively, the specific heat ratio $\gamma$, the increase in pressure per unit increase in temperature at constant density $\hat{\beta}=(\partial P / \partial T)_{V}$, and the adiabatic speed of sound $c_{0}$.

The variables describing the dynamic and thermodynamic states of the gas are the particle velocity $\vec{v}$, the pressure, density, temperature and entropy variations $p, \rho^{\prime}, \tau$ and $s$. A complete set of linearized equations governing small amplitude disturbances of the gas includes [2]:

\section{- the Navier-Stokes equation}

$$
\rho_{0} \frac{\partial \vec{v}}{\partial t}=-\operatorname{grad} p+\operatorname{div} \overline{\bar{\sigma}}_{v}=\operatorname{div} \overline{\bar{\sigma}}
$$

where $\overline{\bar{\sigma}}$ is the tensor associated to the total stress on a fluid particle, and the viscous stress tensor $\overline{\bar{\sigma}}_{v}$ is expressed as function of the volume expansion rate $(\operatorname{div} \vec{v})$ and the shear strain rate tensor $\overline{\bar{\varepsilon}}_{s}$ as follows ( $\overline{\overline{\mathbb{1}}}$ being the identity tensor):

$$
\overline{\bar{\sigma}}_{v}=\eta(\operatorname{div} \vec{v}) \overline{\overline{\mathbb{1}}}+2 \mu \overline{\bar{\varepsilon}}_{s} \quad \text { and } \quad \overline{\bar{\varepsilon}}_{s}=\frac{1}{2}\left[\overline{\overline{\operatorname{grad}}} \vec{v}+(\overline{\overline{\operatorname{grad}}} \vec{v})^{T}\right]-\frac{1}{3}(\operatorname{div} \vec{v}) \overline{\overline{\mathbb{1}}} .
$$

\section{- the conservation of mass equation}

$$
-\operatorname{div} \vec{v}=\frac{1}{\rho_{0}} \frac{\partial \rho^{\prime}}{\partial t}=\frac{\gamma}{\rho_{0} c_{0}^{2}} \frac{\partial}{\partial t}(p-\hat{\beta} \tau)=0,
$$

- the Fourier equation for heat conduction, the heat flow in the gas being $\vec{q}=-\lambda \overrightarrow{\operatorname{grad}} \tau$,

$$
\rho_{0} T_{0} \frac{\partial s}{\partial t}=\rho_{0} C_{P} \frac{\partial \tau}{\partial t}-\frac{\partial p}{\partial t}=-\operatorname{div} \vec{q} .
$$

The solutions of these fundamental equations, associated to adapted boundary conditions, allow us to express the acoustic, velocity and temperature fields in the piezo-resistive microphone. In the following, either an analytical resolution, relying on "classical" quasi-plane waves approximation, or a numerical modelling, by finite element method, are suggested.

\subsection{Analytical modeling, quasi-plane wave approximation}

For the purpose of an analytical modeling of the microphone's acoustic behavior, the microphone is considered as a network of thin rectangle slits coupled to four membranes. Each slit is modeled individually, and then connected to each other by pressure and volume velocity continuity conditions at the interfaces.

In the audio frequency range (frequency $f$ from $20 \mathrm{~Hz}$ to $20 \mathrm{kHz}$ ), the dimensions of the slits constituting the microphone (from 1 to $100 \mu \mathrm{m}$ widths) are small respect to the acoustic wavelengths, but are of the same order as the viscous and thermal boundary layers depths $\delta_{v}=\sqrt{\mu /\left(\pi f \rho_{0}\right)}$ and $\delta_{h}=\sqrt{\lambda /\left(\pi f C_{P} \rho_{0}\right)}$ (Figure 5).

In order to avoid overly intricate formulations, the following appropriate approximations are assumed on the fundamental linear equations (4a-6): $i$. mostly through the boundary layers, 


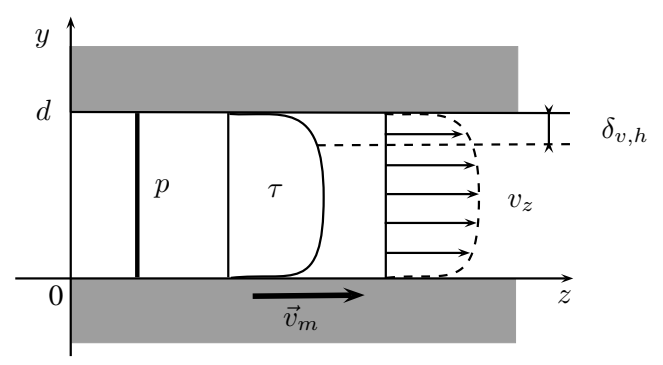

FIGURE 5: Acoustic quasi-plane wave in a rectangle slit.

the component normal to the wall of the acoustic velocity $v_{y}$ is much lower than the tangential component $v_{z}$, ii. the spatial variation of the acoustic velocity is much higher in the normal direction $y$ than in the tangential directions $z$.

According to these assumptions, for an harmonic motion (angular frequency $\omega$ ), the particle velocity, and the pressure and temperature variations in a rectangle slit (width $d$ ) are described by a set of equations, including the mass conservation equation (5), and the following viscous and diffusion equations, at the lowest order of the boundary layers depths $\delta_{v, h}$,

$$
\left(1+\frac{1}{k_{v}^{2}} \frac{\partial^{2}}{\partial y^{2}}\right) v_{z} \approx-\frac{1}{\mathrm{j} \omega \rho_{0}} \frac{\partial p}{\partial z}, \quad \text { and } \quad\left(1+\frac{1}{k_{h}^{2}} \frac{\partial^{2}}{\partial z^{2}}\right) \tau \approx \frac{\gamma-1}{\gamma \hat{\beta}} p
$$

where $k_{v, h}=(1-\mathrm{j}) / \delta_{v, h}$, and $(\gamma-1) /(\gamma \hat{\beta})=1 /\left(\rho_{0} C_{P}\right)$.

The velocity and the temperature fields, subjected to non-slip conditions $\left(v_{y}=0\right.$ and $\left.v_{z}=v=m\right)$ and isothermal conditions $(\tau=0)$ respectively on the boundaries $(y=0$ and $y=d)$, they can be described making use of the mean values over a cross section of the solutions $v_{z}(y, z)$ and $\tau(y, z)$ of equations (7), leading then to the following solutions:

$$
\left\langle v_{z}(z)\right\rangle \approx-\frac{1}{\mathrm{j} \omega \rho_{0}} \frac{\partial}{\partial z} p(z)\left(1-K_{v}\right)+\frac{K_{v}}{2} v_{m}, \quad \text { and } \quad\langle\tau(z)\rangle \approx \frac{\gamma-1}{\hat{\beta} \gamma} p(z)\left(1-K_{h}\right),
$$

where the terms $K_{v, h}=\left\langle\cos \left[k_{v, h}(y-d / 2)\right] / \cos \left(k_{v, h} d / 2\right)\right\rangle=\tan \left(k_{v, h} d / 2\right) /\left(k_{v, h} d / 2\right)$ express the viscous and thermal effects in the boundary layers.

Finally, making use of these expressions in the mean value over a cross section of equation (5) leads to a simple propagation equation for the acoustic pressure $p(z)$ :

$$
\left(\frac{\partial^{2}}{\partial z^{2}}+k_{z}^{2}\right) p=0, \quad \text { with } \quad k_{z}^{2} \approx k_{0}^{2} \frac{1+(\gamma-1) K_{h}}{1-K_{v}} .
$$

Each slit of the planar microphone is then considered as a single propagation domain, associated to a wavenumber $k_{z}$. The acoustic pressure inside each slit is then written as

$$
p(z)=A\left[\exp \left(-\mathrm{j} k_{z} z\right)+B \exp \left(\mathrm{j} k_{z} z\right)\right],
$$

the integration constants $A$ and $B$ being given for each slit by the input and output boundary conditions, and by pressure and volume velocity continuity conditions at the junctions slits, accounting for some diffraction effects at the junctions as simple inertial terms depending on the geometrical configuration of the slits and of the junction [9].

The results obtained from these models generally have a good agreement with experimental results. However, they do not include the coupling between turbulent and diffraction effects at the discontinuities and viscous and thermal effects in the boundary layers, that could be insufficient to model the accurate behavior of the microphone considered here. 


\subsection{Numerical modeling, finite element method}

The finite element modeling procedure presented here is performed according to the method described in reference [8]. An integral formulation is then obtained by $i$. multiplying equations $(4 \mathrm{a}, 5,6)$ by test functions $\left(-\vec{v}^{*}\right), p^{*}$ and $\tau^{*}$ respectively, $i i$. integrating them over the propagation domain $D$ (closed by the surface $S$ with $\vec{n}$ the normal to $S$ outwardly oriented) and summing the integral equations obtained, iii. making use of Green's formula on the terms involving $\left[\vec{v}^{*} .(\operatorname{div} \overline{\bar{\sigma}})\right]$ and $\left(\tau^{*} \operatorname{div} \vec{q}\right)$.

Finally, substituting $\vec{q}$ and (div $\overline{\bar{\sigma}}$ ) by their respective expressions ( $(3.1)$ in the integrals over $D$ leads to the following general weak formulation:

$$
\begin{aligned}
0= & \int_{D} \vec{v}^{*} \cdot\left[-\rho_{0} \frac{\partial \vec{v}}{\partial t}\right] \mathrm{d} D-\int_{D}\left(\overline{\overline{\operatorname{grad}}} \vec{v}^{*}\right): \overline{\bar{\sigma}}_{v} \mathrm{~d} D+\int_{D}\left(\operatorname{div} \vec{v}^{*}\right) p \mathrm{~d} D+\int_{S} \vec{v}^{*} \cdot(\overrightarrow{\bar{\sigma}} \vec{n}) \mathrm{d} S \\
& +\int_{D} \tau^{*}\left[\frac{\rho_{0} C_{P}}{T_{0}} \frac{\partial \tau}{\partial t}-\frac{1}{T_{0}} \frac{\partial p}{\partial t}\right] \mathrm{d} D+\int_{D} \frac{\lambda}{T_{0}}\left(\overrightarrow{\operatorname{grad}} \tau^{*}\right) \cdot(\operatorname{grad} \tau) \mathrm{d} D+\int_{S} \tau^{*} \frac{\vec{q} \cdot \vec{n}}{T_{0}} \mathrm{~d} S \\
& +\int_{D} p^{*}\left[\frac{1}{P_{0}} \frac{\partial p}{\partial t}-\frac{1}{T_{0}} \frac{\partial \tau}{\partial t}\right] \mathrm{d} D+\int_{D} p^{*}(\operatorname{div} \vec{v}) \mathrm{d} D .
\end{aligned}
$$

The two first lines of this integral equation express the shear viscous and thermal diffusion processes in the domain $D$, accounting for the boundary conditions on the walls of the microphone and at its input and output (integrals over surface $S$ ). The last line expresses the coupling between the particle velocity $\vec{v}$, and the temperature and pressure variations $\tau$ and $p$.

The test functions being chosen to satisfy Dirichlet boundary conditions on the walls of the microphone, the integrals over $S$ reduce to integrals over the surfaces $S_{i n}$ and $S_{\text {out }}$ of the input and output holes of the microphone. At the microphone's input and output (open holes), the temperature continuity imposes zero heat rate $(\vec{q} \cdot \vec{n}=0)$. The boundary conditions on $\overline{\bar{\sigma}} \vec{n}$ are imposed by the external acoustic source and the back acoustic load (depending on the practical mounting of the microphone in its package, Fig. 2), that gives $\overline{\bar{\sigma}} \vec{n}=-p_{\text {in }} \vec{n}$ and $\overline{\bar{\sigma}} \vec{n}=-p_{\text {out }} \vec{n}$.

Using a Galerkin approach for discretization, the interpolation functions used for the test functions are identical to the corresponding fields, and the weak formulation (11) leads then to the following symmetric linear system, where the vectors $\mathbf{v}(\omega), \boldsymbol{\tau}(\omega)$ and $\mathbf{p}(\omega)$ gather the nodal values of fields $\vec{v}(\omega), \tau(\omega)$ and $p(\omega)$ respectively:

$$
\left[\begin{array}{ccc}
\mathbf{A}(\omega) & \mathbf{0} & \mathbf{B}(\omega) \\
\mathbf{0} & \mathbf{C}(\omega) & \mathbf{D}(\omega) \\
\mathbf{B}^{T}(\omega) & \mathbf{D}^{T}(\omega) & \mathbf{E}(\omega)
\end{array}\right]\left[\begin{array}{l}
\mathbf{v}(\omega) \\
\boldsymbol{\tau}(\omega) \\
\mathbf{p}(\omega)
\end{array}\right]=\left[\begin{array}{l}
\mathbf{L} \\
\mathbf{0} \\
\mathbf{0}
\end{array}\right] p_{i n}(\omega)
$$

where the load vector $\mathbf{L}$ results from the surface integrals discretization, and the symmetric matrices $\mathbf{A}, \mathbf{C}, \mathbf{E}$ and coupling matrices $\mathbf{B}, \mathbf{D}$ result from the discretization of the terms:

$$
\begin{aligned}
& \mathbf{A}(\omega) \equiv \int_{D} \eta\left(\operatorname{div} \vec{v}^{*}\right)(\operatorname{div} \vec{v})+2 \mu\left(\overline{\bar{\varepsilon}}_{s}^{*}: \overline{\bar{\varepsilon}}_{s}\right)+\mathrm{j} \omega \rho_{0} \vec{v}^{*} \cdot \vec{v} \mathrm{~d} D, \\
& \mathbf{C}(\omega) \equiv-\int_{D} \frac{\lambda}{T_{0}}\left(\overrightarrow{g r a d} \tau^{*}\right) \cdot(\operatorname{grad} \tau)+\mathrm{j} \omega \frac{\rho_{0} C_{P}}{T_{0}} \tau^{*} \tau \mathrm{d} D, \quad \mathbf{E}(\omega) \equiv \int_{D} \frac{\mathrm{j} \omega}{P_{0}} p^{*} p \mathrm{~d} D, \\
& \mathbf{B}(\omega) \equiv-\int_{D} p \operatorname{div} \vec{v}^{*} \mathrm{~d} D \quad \text { and } \quad \mathbf{B}^{T}(\omega) \equiv-\int_{D} p^{*} \operatorname{div} \vec{v} \mathrm{~d} D \\
& \mathbf{D}(\omega) \equiv \int_{D} \frac{\mathrm{j} \omega}{T_{0}} \tau^{*} p \mathrm{~d} D \quad \text { and } \quad \mathbf{D}^{T}(\omega) \equiv \int_{D} \frac{\mathrm{j} \omega}{T_{0}} p^{*} \tau \mathrm{d} D
\end{aligned}
$$

\section{STUDY OF A MICROPHONE'S ELEMENTAL PART: LIMITS OF THE MODELS}

In order to check the validity of the two approaches (analytical and numerical) carried out to model the acoustic behavior of a planar microphone, the results obtained from them for a simple 
structure are compared in this section. This structure is composed of two "large" input and output rectangle slits, coupled to a membrane, and to two "thin" slits above and under the membrane (Fig. 6). This system represents an elemental part of the total microphone's structure (Fig. 1a). As a first step of this validation work, the membrane's vibroacoustic behavior is not considered, and the membrane is assumed to be a perfectly rigid wall.

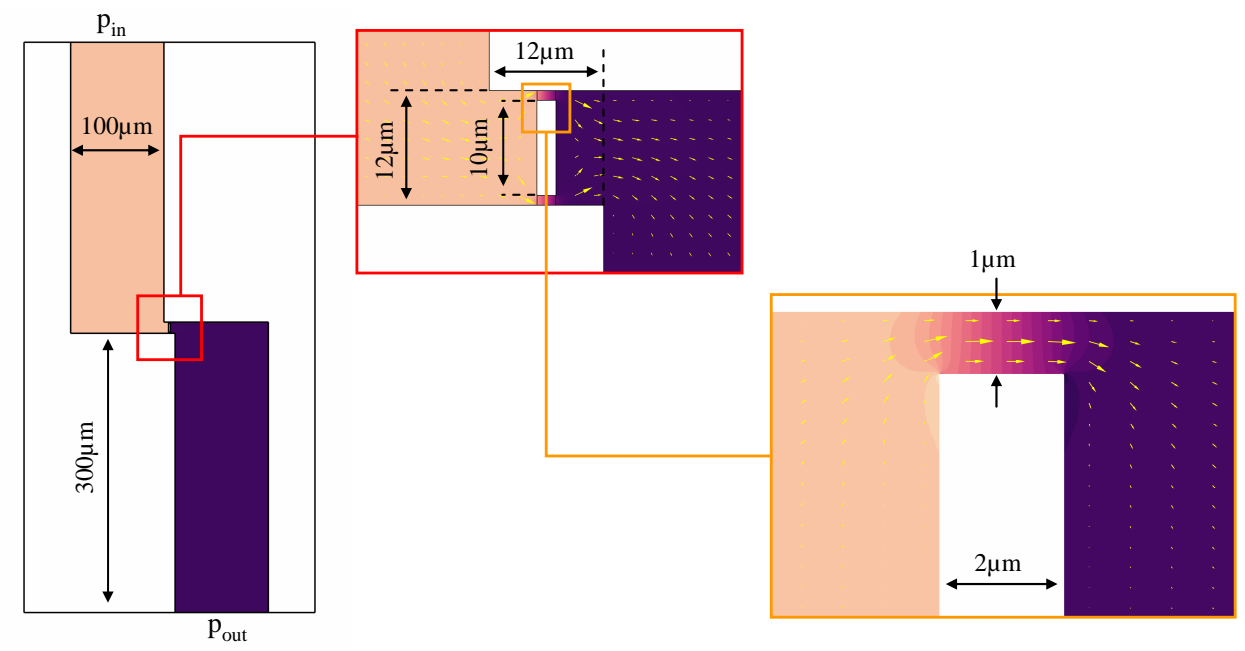

FIGURE 6: Side view of the pressure and particle velocity fields in a thin slits network, in atmospheric conditions, at $1 \mathrm{kHz}$, calculated from the numerical model (FEM) (§ 3.3)

The spatial repartition of the acoustic and particle velocity fields (Fig. 6) show that the quasi-plane wave approximation (Fig. 5) is adapted to describe the acoustic field inside each slit. Some perturbations to the acoustic and velocity fields are visible at the junctions and angles.

The quantity of interest for further determination of microphone's pressure sensitivity, defined in eq. (3), is the transfer function $p_{m} / p_{i n}$, namely the ratio of the total acoustic pressure $p_{m}$ applied to the membrane to the acoustic pressure $p_{i n}$ at the input of the system (Fig. 7).
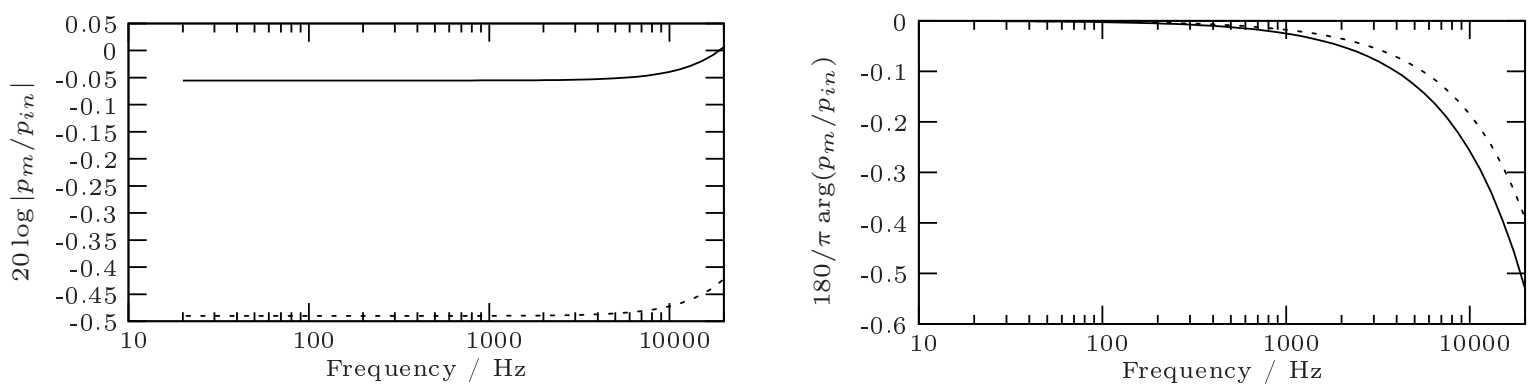

FigURE 7: Transfer function $p_{m} / p_{\text {in }}$ (module and phase) of the acoustic system on Figure 6, calculated from the analytical model $\S 3.2$ (solid lines) and from the numerical model $\S 3.3$. (dashed lines).

These results show that the attenuation of the acoustic pressure between the input of the system and the membrane is very small, and the system's frequency response is flat in the frequency range of interest. As expected, the analytical and numerical models lead to coherent results. The discrepancies between the results derived from the two approaches are small but significant, since they reach a maximum of $0.4 \mathrm{~dB}$, whereas the maximum uncertainties required on the model should be the order of few $0.1 \mathrm{~dB}$. On one hand, these discrepancies would be due to the fact that the analytical modeling does not include the effects of turbulent perturbations at the discontinuities. On the other hand, there are also some numerical errors at the discontinuities, that remains unevaluated, and will be the object of further studies. 


\section{Conclusion}

The first theoretical results obtained from two different modeling procedures seem encouraging about planar microphones performances in terms of sensitivity and resolution. Moreover, these two theoretical approaches allow us to evidence effects of viscous turbulent perturbations at discontinuities, that can be significant in micro and nano acoustic systems.

Through encouraging, these first results have to be completed by $i$. optimizing the discretization procedure of the finite element method carried out here especially close to the angles, $i$. introducing the membranes vibroacoustic behavior coupled to the acoustic field in the numerical modeling, iii. achieving an experimental validation of the analytical and numerical models of miniature acoustic systems presented here [10].

\section{ACKNOWLEDGMents}

This work is supported by the Agence Nationale de la Recherche (ANR) as part of the MADNEMS project (ANR-11-NANO-026).

\section{REFERENCES}

[1] A. Walther, M. Savoye, G. Jourdan, P. Renaux, F. Souchon, P. Robert, C. Le Blanc, N. Delorme, O. Gigan, and C. Lejuste, "3 axis gyroscope with Si nanogauge piezo-resistive detection”, in Proc. IEEE MEMS (2012).

[2] M. Bruneau and T. Scelo (translator and contributor), Fundamentals of Acoustics (ISTE, London) (2006).

[3] T. Le Van Suu, S. Durand, and M. Bruneau, "On the modelling of clamped plates loaded by a squeeze fluid film: application to miniaturised sensors", Acta Acust. united Ac. 96, 923-935 (2010).

[4] T. Lavergne, S. Durand, M. Bruneau, and N. Joly, "Dynamic behavior of the circular membrane of an electrostatic microphone: Effect of holes in the backing electrode", J. Acoust. Soc. Am. 128, 3459-3477 (2010).

[5] C. Karra and M. Ben Tahar, "Boundary element analysis of vibratory behaviour of a membrane coupled to a thermal conductor layer fluid”, C. R. Académie des Sciences, Série II B - Mécanique Physique Astronomie 326, 733-739 (1998).

[6] H. Inayat Hussain and J.-L. Guyader, "Sound radiation of plates in thermoviscous media", Acta Acust. united Ac. 95, 997-1005 (2009).

[7] N. Joly, "Finite element modeling of thermoviscous acoustics on adapted anisotropic meshes: Implementation of the particle velocity and temperature variation formulation", Acta Acust. united Ac. 96, 397 (2010).

[8] W. R. Kampinga, Y. H. Wijnant, and A. de Boer, "An efficient finite element model for viscothermal acoustics”, Acta Acust. united Ac. 97, 618-631 (2011).

[9] J. Kergomard and A. Garcia, "Simple discontinuities in acoustic waveguides at low frequencies: critical analysis and formulae", J. Sound Vib. 114, 465-479 (1987).

[10] D. Rodrigues, C. Guianvarc'h, J.-N. Durocher, M. Bruneau, and A.-M. Bruneau, "A method to measure and interpret input impedance of small acoustic components", J. Sound Vib. 315, 890-910 (2008). 